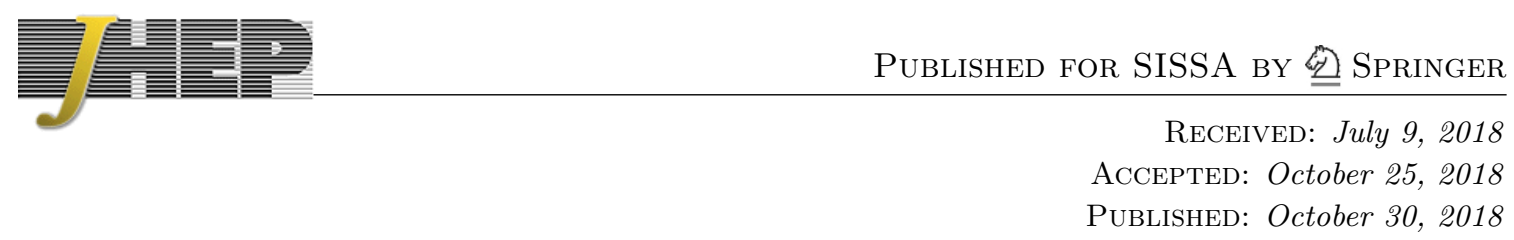

\title{
Clockwork axions in cosmology. Is chromonatural inflation chrononatural?
}

\author{
Prateek Agrawal, ${ }^{a}$ JiJi Fan $^{b}$ and Matthew Reece ${ }^{a}$ \\ ${ }^{a}$ Department of Physics, Harvard University, \\ 17 Oxford Street, Cambridge, MA 02138, U.S.A. \\ ${ }^{b}$ Department of Physics, Brown University, \\ 184 Hope Street, Providence, RI 02912, U.S.A. \\ E-mail: prateek@fnal.gov, jijifan1982@gmail.com, \\ mreece@physics.harvard.edu
}

ABSTRACT: Many cosmological models rely on large couplings of axions to gauge fields. Examples include theories of magnetogenesis, inflation on a steep potential, chiral gravitational waves, and chromonatural inflation. Such theories require a mismatch between the axion field range and the mass scale appearing in the $a F \widetilde{F}$ coupling. This mismatch suggests an underlying monodromy, with the axion winding around its fundamental period a large number of times. We investigate the extent to which this integer can be explained as a product of smaller integers in a UV completion: in the parlance of our times, can the theory be "clockworked"? We argue that a clockwork construction producing a potential $\mu^{4} \cos \left(\frac{a}{j F_{a}}\right)$ for an axion of fundamental period $F_{a}$ will obey the constraint $\mu<F_{a}$. For some applications, including chromonatural inflation with sub-Planckian field range, this constraint obstructs a clockwork UV completion. Alternative routes to a large coupling include fields of large charge (an approach limited by strong coupling) or kinetic mixing (requiring a lighter axion). Our results suggest that completions of axion cosmologies that explain the large parameter in the theory potentially alter the phenomenological predictions of the model.

Keywords: Anomalies in Field and String Theories, Cosmology of Theories beyond the SM

ArXiv EPrint: 1806.09621 


\section{Contents}

1 Introduction: large couplings in axion cosmology 1

2 Mechanisms to generate large axion couplings 4

2.1 Vector-like matter with large charges 4

2.2 Kinetic mixing 4

2.3 Non-compact fields 5

3 Theoretical constraints on alignment and clockwork mechanisms 5

$\begin{array}{ll}3.1 & \text { Unitarity constraint on single axion model }\end{array}$

$\begin{array}{lll}3.2 & \text { Two-site alignment model } & 7\end{array}$

3.3 N-site clockwork 8

4 A clockworkable example: Anber-Sorbo inflation $\quad 9$

$\begin{array}{lll}5 & \text { Chromonatural inflation } & 11\end{array}$

6 Conclusions $\quad 15$

$\begin{array}{ll}\text { A How confining models ensure } \mu<f_{a} & 15\end{array}$

\section{Introduction: large couplings in axion cosmology}

A large set of cosmological models rely on a (pseudo)scalar field coupled to an $F \widetilde{F}$ term for a gauge field or an $R \widetilde{R}$ term for gravity. Applications include the generation of primordial magnetic fields [1-3]; dissipation allowing for inflation in steep potentials [4-6]; baryo- or leptogenesis [7]; chromonatural inflation [8-11]; production of chiral gravitational waves during inflation [12-18]; preheating [19]; decreasing the abundance of QCD axion dark matter $[20,21]$ and providing alternative dissipation mechanisms [22-24] in relaxion cosmology [25].

Some of these theories are particularly interesting because they allow qualitatively new phenomena compared to conventional theories. For instance, the usual manner in which inflation can produce a possibly observable tensor-to-scalar ratio is large-field inflation, that is, if the inflaton field range is larger than the Planck scale. This is the so-called "Lyth bound" [26]. Theories like chromonatural inflation can produce a large signal from chiral primordial gravitational waves, even with a small field range, thus evading the Lyth bound. The central element is the presence of gauge fields which are constantly replenished by the rolling inflaton field. These gauge fields have tensor fluctuations which are not related to scalar fluctuations in the same way as in usual slow-roll inflation. Another interesting result 
is that axion couplings to gauge fields, by providing a source of dissipation other than Hubble friction, can allow inflation in steep potentials that fail the usual slow-roll criteria [4].

Both of these examples have heightened interest due to various "Swampland" conjectures about what is possible in quantum gravity [27-29]. For example, theories of large-field inflation run into tension with the failure of explicit searches for super-Planckian field ranges in string theory [30-36], with the Weak Gravity Conjecture [28, 37-43], and with more general Swampland arguments about difficulties with super-Planckian field ranges [29, 44-51]. These difficulties make chromonatural inflation particularly interesting as a theory that can generate a large tensor signal while possibly evading such constraints. More recently, some authors have speculated that there are no consistent de Sitter vacua in quantum gravity [52-56] (also see [57, 58]). An even stronger statement has been suggested: there is no region of the scalar potential in quantum gravity for which $V>0$ and $M_{\mathrm{pl}}\left|\partial_{\phi} V\right| / V \ll 1$, putting tension on the slow-roll inflation paradigm [59,60]. This makes inflating in steep potentials through dissipation an even more interesting possibility, as it evades such hypothetical (but speculative) Swampland bounds. For these reasons, it is very interesting to consider the model-building of these theories in more detail, to assess how plausible UV completions may be.

A common feature for the cosmological models mentioned above is an axion-gauge field coupling that is parametrically large relative to the field range of the axion. An axion is a periodic field, $a \cong a+2 \pi F_{a}$ with $F_{a}$ being the fundamental period. A typical Lagrangian in these models takes the form

$$
\mathcal{L} \supset \mu^{4} \cos \left(\frac{a}{j F_{a}}\right)+k \frac{\alpha}{8 \pi} \frac{a}{F_{a}} F_{\mu \nu} \widetilde{F}^{\mu \nu},
$$

where the first term is the effective potential of the axion generated (for example) by nonperturbative dynamics of some confining gauge group with a confinement scale $\mu$. The coefficient $j$ appears to violate this shift symmetry, but if it is an integer it can arise via monodromy, i.e. a potential with multiple branches [61-63]. In the second term, $F_{\mu \nu}$ is the field strength of some other Abelian or non-Abelian gauge group, which will play different roles depending on the model. The coefficient $k$ must be an integer due to the axion shift symmetry. The effective field range of the axion is then

$$
f_{a} \equiv j F_{a} .
$$

Note that when gauge fields are canonically normalized, the presence of the factor of $\alpha=\frac{g^{2}}{4 \pi}$ with $g$ being the gauge coupling is required for consistency with the discrete shift symmetry of the axion. Unsurprisingly, the same factor appears in the $\theta$-term for the gauge theory. Quite a few phenomenological applications in the cosmological models simply write the axion-gauge field couplings as $\frac{\lambda}{f_{a}} a F_{\mu \nu} \widetilde{F}^{\mu \nu}$ without explicitly writing the coupling strength. ${ }^{1}$ These applications typically require the dimensionless coefficient $\lambda \gg 1$. In our notation in eq. (1.1), this requirement is translated to

$$
k j \alpha \gg 1 .
$$

\footnotetext{
${ }^{1}$ Be aware of notation: some literature used $\alpha$ instead of $\lambda$ to denote the overall axion-gauge field coupling, but this $\alpha$ is not $g^{2} /(4 \pi)$.
} 
This factor of $\alpha=g^{2} /(4 \pi)$ has not been emphasized in the literature of many cosmological models. In perturbative models, $\alpha \lesssim 0.1$ when the gauge coupling is $\sim 1$. Sometimes there are further constraints on the size of the gauge coupling and $\alpha$ has to be smaller. Taking $\alpha$ into account, an enhanced axion-gauge field coupling usually requires a huge value for $k \times j$, which could be orders of magnitude larger than the number quoted in the literature. While there are ways of getting enhanced couplings of axions to gauge fields [64, 65], these mechanisms are subject to simple theoretical constraints. It is thus highly non-trivial and interesting to investigate whether and how to get the large axion-gauge field coupling needed for interesting cosmological dynamics.

We see from the discussion above that there are two enhancement factors, an enhanced coupling $k$ and an enhanced field range $j$. There are three possible types of mechanisms to generate a large coupling between the compact axion field and gauge fields. The first two enhance the coupling via increasing $k$, while the third enhances the field range.

- Inclusion of matter fields such as vector-like fermions with large charges under the gauge group or large PQ charge;

- Kinetic mixing between multiple axions [66];

- Two axion Kim-Nilles-Peloso (KNP) alignment [67] or its generalization to models with more than two axions (clockwork models $[68,69]$ ).

In the QCD axion context, the three mechanisms and their qualitative enhancement factors have been studied in ref. [65]. It is important to keep in mind that all the mechanisms are subject to different theoretical constraints and may not generate arbitrarily large couplings. In particular, we will argue that

$$
\begin{aligned}
\text { Large charges } & \Rightarrow k \lesssim \alpha^{-1}, \\
\text { Clockwork } & \Rightarrow j \lesssim f_{a} / \mu .
\end{aligned}
$$

The kinetic mixing case potentially evades the bound on $k$, but this requires a lighter axion field to be present in the EFT. Together, these considerations can significantly constrain the available options for UV completing an effective field theory of axions coupled to gauge fields.

The models we study involve enhanced couplings of an axion-like particle to the gauge field, which requires the presence of fermions charged under the gauge group close to the scale suppressing the operator. The cosmology of these models additionally involves the production of large gauge fields. An interesting possible consequence is that these large gauge fields lead to non-perturbative instabilities via Schwinger pair-production of charged fermions or non-Abelian gauge fields [23, 24, 70-74]. Clockwork theories of multiple axions also have interesting networks of topological defects that can affect the cosmology [75]. A detailed study of these phenomena is beyond the scope of this paper.

In section 2 we summarize the enhancement with mechanisms that rely on matter with large charges or kinetic mixing, and the associated theoretical constraints. The mechanism that could lead to the most significant enhancement is the clockwork mechanism. We will 
discuss the constraints on it and alignment model in detail in section 3. Section 4 discusses large couplings in the Anber-Sorbo inflation model, which could be clockworked. Section 5 discusses the chromonatural inflation model, in which the necessary enhancement for the axion-gauge field coupling is so large that even the clockwork mechanism (which is the most efficient way to generate an exponentially large coupling) could not work unless the inflaton field range is super-Planckian. We offer concluding remarks in section 6 .

\section{Mechanisms to generate large axion couplings}

In this section, we will discuss issues generating large axion couplings to gauge fields via a large value of $k$, which may be obtained by integrating out matter with large charges or through kinetic mixing.

\subsection{Vector-like matter with large charges}

Let us consider the following interaction

$$
\frac{k \alpha}{8 \pi F_{a}} a F \widetilde{F}
$$

Here, $k$ is an anomaly coefficient that depends on the gauge and PQ charges (and the number of flavors) of fermions integrated out at the scale $F_{a}$. As discussed in appendix $\mathrm{C}$ of ref. [65], larger $\mathrm{PQ}$ charges $q_{\mathrm{PQ}}$ require small fermion masses $\propto\left(F_{a} / \Lambda\right)^{q_{\mathrm{PQ}}}$, and it is not possible to get a very large enhancement by increasing $P Q$ charges alone. We will not pursue the large PQ charge case further and assume the PQ charge is 1.

We briefly recall the problem with large gauge charges. We imagine that the axion interaction is generated by $N_{f}$ fermions $Q$ as in the KSVZ axion model [76, 77]:

$$
\mathcal{L}=\frac{N_{f} I_{2}(Q) \alpha}{4 \pi F_{a}} \phi F_{\mu \nu}^{a} \widetilde{F}^{a, \mu \nu}
$$

where $I_{2}(Q)$ is the Dynkin index of the representation. However, requiring that the gauge theory is perturbative at the scale of the PQ breaking requires

$$
N_{f} I_{2}(Q) \alpha \lesssim 1
$$

Therefore, $k \alpha \lesssim 1$.

In these models the fermions get a mass from the spontaneous breaking of the PQ symmetry, so that $m_{f} \lesssim F_{a}$.

\subsection{Kinetic mixing}

Kinetic mixing of two axions can contribute to enhanced couplings to a gauge group [20, 78, 79]. To review the basic mechanism, consider the following simplified Lagrangian where the cosmologically evolving axion $a$ kinetically mixes with a lighter axion $b$,

$$
\mathcal{L}=\frac{1}{2}(\partial a)^{2}+\frac{1}{2}(\partial b)^{2}+\epsilon\left(\partial_{\mu} a\right)\left(\partial^{\mu} b\right)+\mu^{4} \cos \frac{a}{F_{a}}+\frac{\alpha}{8 \pi F_{b}} b F_{a}^{\mu \nu} \widetilde{F}^{a, \mu \nu}
$$


The axion $a$ inherits the coupling

$$
\frac{\epsilon F_{a}}{F_{b}} \frac{\alpha}{8 \pi F_{a}} a F_{a}^{\mu \nu} \widetilde{F}^{a, \mu \nu}
$$

so that for $\epsilon F_{a} / F_{b}=k \gg 1$, the couplings of $a$ can be enhanced.

However, we see that there is a coupling of axion $b$ to the gauge bosons with a very small value of $F_{b}$. Therefore, the price for this enhancement are states charged under the gauge group which are much lighter than $F_{a}$. The presence of such very light charged fermions can significantly alter the phenomenology, e.g. due to Schwinger pair production.

\subsection{Non-compact fields}

While most of the discussions in the paper will focus on the case of a compact axion field, the issue of generating a large coupling can be studied more generally even beyond theories of compact axions. For instance, let us consider the following theory without a fundamental shift symmetry,

$$
-\frac{1}{2} m^{2} \phi^{2}-\frac{c_{4}}{4 !} \frac{m^{2}}{f^{2}} \phi^{4}+\frac{k \alpha}{f} \phi F \widetilde{F}+\cdots,
$$

with $f$ being the field range of $\phi$. The dimensionless coefficient $c_{4}$ is order-one in general. Now, suppose that we generate the $\phi F \widetilde{F}$ coupling by integrating out a Dirac fermion beginning with the renormalizable Lagrangian

$$
m_{Q} \bar{Q} Q+\mathrm{i} y \phi \bar{Q} \gamma^{5} Q
$$

In this case, integrating out the fermion $Q$ will generate an effective coupling of order

$$
\frac{g^{2} I_{2}(Q)}{16 \pi^{2}} \arg \left(m_{Q}+\mathrm{i} y \phi\right) F \widetilde{F} \lesssim \frac{g^{2} I_{2}(Q)}{16 \pi^{2}} \frac{\phi}{f} F \widetilde{F}
$$

with $I_{2}(Q)$ the Dynkin index of the representation of $Q$ under the gauge group. In the second step we have assumed that $m_{Q} \gtrsim y \phi \sim y f$, since the value of $\phi$ contributes to the mass of $Q$. Perturbativity of the theory requires that $\alpha I_{2}(Q) \lesssim 1$. As a result, we see that again it is difficult to explain the large $\phi F \widetilde{F}$ coupling relative to $1 / f$ (with $f$ the field range) without a UV completion that contains more structure. This motivates thinking about mechanisms like alignment or clockwork, which we will discuss more in detail below, even though we have not assumed that $\phi$ is a fundamentally compact field. In particular, here $f$ was merely set by the range of values $\phi$ traverses, not by its period.

\section{Theoretical constraints on alignment and clockwork mechanisms}

In this section, we discuss a simple theoretical constraint on using the alignment or the clockwork mechanism to generate a large axion coupling to gauge fields. The constraint is, in terms of parameters in the effective Lagrangian of the axion given by eq. (1.1)

$$
\mu \lesssim F_{a}
$$


We want to emphasize the right hand side is the fundamental period of the axion, instead of the effective field range, which could be significantly larger in monodromy models including alignment and clockwork models. The constraint is rooted in the unitarity constraint on a single axion, which will be described in section 3.1. Then we will prove it in the two-axion alignment and multi-axion clockwork model.

\subsection{Unitarity constraint on single axion model}

Our argument relies fundamentally on the following claim: in any effective theory containing the potential for the single axion

$$
V(a)=\mu^{4}\left[1-\cos \left(\frac{a}{f_{a}}\right)\right],
$$

we must have $\mu \lesssim f_{a}$. This follows simply from perturbative unitarity. Expanding around the minimum of the potential, we have

$$
V(a)=\frac{1}{2} \frac{\mu^{4}}{f_{a}^{2}} a^{2}-\frac{1}{4 !} \frac{\mu^{4}}{f_{a}^{4}} a^{4}+\frac{1}{6 !} \frac{\mu^{4}}{f_{a}^{6}} a^{6}+\cdots,
$$

so the low-energy amplitude for $a a \rightarrow a a$ scattering behaves as

$$
\mathcal{M} \approx\left(\frac{\mu}{f_{a}}\right)^{4}\left(1-\frac{c}{16 \pi^{2}} \frac{\Lambda_{\mathrm{UV}}^{2}}{f_{a}^{2}}+\cdots\right)
$$

with $\Lambda_{\mathrm{UV}}$ an ultraviolet cutoff on loops computed with the effective Lagrangian and $c$ some scheme-dependent order-one constant. The first term is the tree-level amplitude, the second comes from beginning with a six-point vertex and sewing up two lines to form a 1-loop diagram, and a series of additional terms appearing with further powers of $\Lambda_{\mathrm{UV}} / f$ will arise from higher-point couplings.

Unitarity requires that the amplitude $\mathcal{M}$ be bounded: specifically, the $\ell=0$ partial wave amplitude $a_{0}$ should satisfy $\left|\operatorname{Re} a_{0}\right|<\frac{1}{2}$. In perturbation theory, $a_{0} \approx-\frac{\mathcal{M}}{16 \pi}$. Thus, in order for perturbation theory to be approximately reliable we must require

$$
\begin{aligned}
\left(\frac{\mu}{f_{a}}\right)^{4} & \lesssim 8 \pi \Rightarrow \mu \lesssim 2 f_{a}, \\
\Lambda_{\mathrm{UV}} & \lesssim 4 \pi f_{a} .
\end{aligned}
$$

This already places some constraints on scenarios discussed in the literature; for example, the gauge-flation trajectory of [80] is discussed for a parameter point with $\mu=4 f_{a}=$ $4 \times 10^{-2} M_{\mathrm{pl}}$. In this case, treating the theory using the classical equations of motion in a cosine potential is not expected to be valid, because unitarity requires large corrections to answers computed perturbatively from the Lagrangian.

One could ask how the constraint $\mu<f_{a}$ is enforced in UV completions; for example, we can write a renormalizable theory breaking Peccei-Quinn symmetry at some scale $f_{a}$ and with confinement at some other scale $\Lambda$, and a priori these appear to be independently adjustable parameters. However, if one tries to build a model with $\mu \gg f_{a}$, one always 
finds that confinement has important effects on the PQ-breaking dynamics that ensure the bound is respected. Simple examples illustrating this point are discussed in appendix A.

Let us pause here to discuss a small technical subtlety that in the end has no impact on our arguments. Depending on how the cosine potential (3.2) is generated, there may already be a monodromy present. For instance, confinement in an $\mathrm{SU}(n)$ Yang-Mills theory is expected to generate a potential with $n$ branches of the form $n^{2} E(\theta / n)$, where $E$ is a function of period $2 \pi n$ despite the fact that $\theta$ has fundamental period $2 \pi$ [61, 62]. The axion potential generated by confinement can be taken schematically to be of the form (3.2) with $\mu^{4} \sim n^{2} \Lambda_{\text {conf }}^{4}$ and $f_{a}=n F_{a}$, with $F_{a}$ the fundamental axion period. However, we are not interested in theories with unexplained, parametrically large $n$ (requiring a large number of fundamental fields and implying a UV cutoff $\left.\lesssim M_{\mathrm{pl}} / n\right)$. We will focus on small$n$ gauge groups and the possibility of generating monodromy through products of smaller integers. As a result, we will suppress the factor of $n$ and take the confinement potential to be $\mu^{4} \cos (a / F)$, rather than $\mu^{4} \cos (a /(n F))$, below. Restoring factors of $n$ will change our final conclusion only by an order-one factor provided all the $n$ 's are themselves order-one. In particular, the main argument will rely on the statement that a potential of the form

$$
\mu_{i+1}^{4} \cos \left[\frac{1}{n_{i+1}}\left(\frac{N_{i} a_{i}}{F_{i}}+\frac{a_{i+1}}{F_{i+1}}\right)\right]
$$

serves to set $a_{i+1}=-N_{i} a_{i} \frac{F_{i+1}}{F_{i}}$, a fact which is independent of the $n_{i+1}$ factor.

\section{$3.2 \quad$ Two-site alignment model}

It is easiest to demonstrate the crucial constraint in eq. (3.1) using the simple two-site alignment model that could enhance the axion coupling. Subsequent to the original work of Kim, Nilles, and Peloso [67], such models have been studied extensively in e.g. [81-84]. The model is given by

$$
\mathcal{L}=\frac{\alpha_{s ; 1}}{8 \pi F_{1}} a_{1} G_{1} \widetilde{G}_{1}+\frac{\alpha_{s ; 2}}{8 \pi}\left(\frac{N a_{1}}{F_{1}}+\frac{a_{2}}{F_{2}}\right) G_{2} \widetilde{G}_{2}+\frac{\alpha}{8 \pi F_{2}} a_{2} F \widetilde{F},
$$

where $G_{1}, G_{2}$ are the field strengths of two heavy confining gauge groups. $N>1$ is an integer and is usually some group theoretical factor in a full model (one full model based on KSVZ fermions could be found in ref. [65]). $F$ is the field strength of a gauged U(1) (which is not necessarily our electromagnetic $\mathrm{U}(1)$ ). It is straightforward to generalize the discussion for $\mathrm{U}(1)$ to that of a non-Abelian gauge field. $\alpha_{s ; 1}=g_{s ; 1}^{2} /(4 \pi), \alpha_{s ; 2}=g_{s ; 2}^{2} /(4 \pi)$, $\alpha=g^{2} /(4 \pi)$ are the coupling strengths of the two confining gauge groups and $\mathrm{U}(1)$ respectively with $g_{s ; 1}, g_{s ; 2}, g$ corresponding gauge couplings. Below the confinement scales, the effective potential of the two axions is

$$
V\left(a_{1}, a_{2}\right)=\mu_{1}^{4} \cos \left(\frac{a_{1}}{F_{1}}\right)+\mu_{2}^{4} \cos \left(\frac{N a_{1}}{F_{1}}+\frac{a_{2}}{F_{2}}\right) .
$$

We need

$$
\mu_{1}<\mu_{2} \lesssim F_{2} .
$$


The first inequality between the two confinement scales $\mu_{1}$ and $\mu_{2}$ is needed for enhancing the light axion coupling to photons. If $\mu_{1}<\mu_{2}$, the heavy axion is

$$
\frac{N a_{1}}{F_{1}}+\frac{a_{2}}{F_{2}},
$$

up to a normalization factor. Integrating out the heavy axion by setting the heavy axion field to be zero, we have the effective Lagrangian for the light axion, which is mostly $a_{2}$ :

$$
\mathcal{L}\left(a_{2}\right)=\mu_{1}^{4} \cos \left(\frac{a_{2}}{N F_{2}}\right)+\frac{\alpha}{8 \pi F_{2}} a_{2} F \widetilde{F} .
$$

Mapping onto eq. (1.1), we have

$$
\mu=\mu_{1}, \quad F_{a}=F_{2}, \quad j=N, \quad f_{a}=N F_{2}, \quad \text { and } \quad k=1 .
$$

To get an enhanced axion-gauge field coupling, eq. (1.3) tells us that $N \alpha \gg 1$.

The second inequality between $\mu_{2}$ and $F_{2}$ is due to the fact that otherwise, the axion quartic coupling would be $\gg 1$ and violates perturbative unitarity, as discussed in the previous section. Another way to understand it is that if $\mu_{2}>F_{2}$, then confinement would break the PQ symmetry first, as discussed in examples in appendix A. The axion potential is then modified and $F_{2}$ in eq. (3.9) should be replaced by $\mu_{2}$. These two inequalities are combined to give us the constraint in eq. (3.1), which in this case is simply $\mu_{1}<F_{2}$.

In this example, we can derive a separate and even stronger bound: $\mu_{1}<\mu_{2} \lesssim F_{1} / N$, again following from unitarity using the second cosine term. If $F_{1} \sim F_{2}$, this is a much stronger bound. In fact, this bound is weaker only when $F_{1}>N F_{2}$ and we have already introduced a large hierarchy of decay constants at the outset, so that alignment is not helping. However, in the clockwork case below the integer $N$ will be a smaller number and there is little difference between the two bounds.

\subsection{N-site clockwork}

The argument for two-axion alignment model could be generalized to $\mathrm{N}$-site clockwork model. This could be mostly easily checked in the confinement tower model [65] (a supersymmetric version of which appeared earlier in [68]; also see [85]). Consider $n$ confining gauge groups $\mathrm{SU}\left(n_{i}\right)$ with field strengths labeled by $G_{i}, i=1,2, \cdots n$ with a Lagrangian

$$
\mathcal{L}=\sum_{i=1}^{n-1} \frac{\alpha_{s ; i+1}}{8 \pi}\left(\frac{N_{i} a_{i}}{F_{i}}+\frac{a_{i+1}}{F_{i+1}}\right) G_{i+1} \widetilde{G}_{i+1}+\frac{\alpha_{s ; 1}}{8 \pi F_{1}} a_{1} G_{1} \widetilde{G}_{1}+\frac{\alpha}{8 \pi F_{n}} a_{n} F \widetilde{F},
$$

where $N_{i} \geq 1$ 's are integers. The potential of the $n$ axions is given by

$$
V\left(a_{i}\right)=\sum_{i=1}^{n-1} \mu_{i+1}^{4} \cos \left(\frac{N_{i} a_{i}}{F_{i}}+\frac{a_{i+1}}{F_{i+1}}\right)+\mu_{1}^{4} \cos \left(\frac{a_{1}}{F_{1}}\right) .
$$

Note that this potential holds in general clockwork models such as the original model based on a number of scalars with particular quartic couplings. ${ }^{2}$

\footnotetext{
${ }^{2}$ In the original model, there are $n$ complex scalars, $\phi_{i}, i=1,2, \cdots n$ with quartic couplings $\phi_{i}^{3} \phi_{i+1}^{\dagger}+$ h.c. Every scalar obtains a vacuum expectation value $\left\langle\phi_{i}\right\rangle$. Their angular modes become the axions with a potential in eq. (3.15), in which all the $N_{i}=3$ and $F_{i}=\left\langle\phi_{i}\right\rangle$.
} 
To enhance the couplings of the lightest axion (which is mostly $a_{n}$ ) to photons, we need

$$
\mu_{1}<\mu_{2,3, \cdots, n}, \mu_{n}<F_{n} .
$$

After integrating out the heavy axions by setting

$$
\frac{N_{i} a_{i}}{F_{i}}+\frac{a_{i+1}}{F_{i+1}}=0, \quad i=1,2, \cdots n-1,
$$

we have the effective Lagrangian of the lightest axion $a_{n}$,

$$
\mathcal{L}_{\text {eff }}=\frac{\alpha_{s ; 1}}{8 \pi\left(\prod_{i=1}^{n-1} N_{i}\right) F_{n}} a_{n} H_{1} \widetilde{H}_{1}+\frac{\alpha}{8 \pi F_{n}} a_{n} F \widetilde{F} .
$$

Mapping onto eq. (1.1), we have

$$
\mu=\mu_{1}, \quad F_{a}=F_{n}, \quad j=\prod_{i=1}^{n-1} N_{i}, \quad f_{a}=\left(\prod_{i=1}^{n-1} N_{i}\right) F_{n}, \quad \text { and } \quad k=1 .
$$

Again the inequalities in eq. (3.16) are reduced to eq. (3.1).

In this case we can derive slightly stronger bounds like $\mu<F_{a} / N_{n-1}$, but if we take the $N_{i}$ to be order-one numbers this is only a modestly more stringent constraint.

Before moving on to our main examples, let us briefly remark on the original relaxion model [25]. In this case the potential is of the form $g M^{2} \phi+g^{2} \phi^{2}+\cdots$, where $g$ is a tiny mass scale reflecting small breaking of the $\phi$ shift symmetry and $M$ is a UV cutoff. This is an expansion in $\phi / f_{\text {large }}$ where $f_{\text {large }} \sim M^{2} / g$. From this we read off that the analogue of $\mu^{4}$ is $g M^{2} f_{\text {large }} \sim M^{4}$. If we try to complete the model with clockwork to explain why $f_{\text {large }} \gg F$, with $F$ the scale appearing in the $\Lambda(h)^{4} \cos (\phi / F)$ term, then eq. (3.1) becomes $M<F$. This constraint is respected in the models of [25]. We have not explored to what extent this remains true in the large literature of variations on the model.

\section{A clockworkable example: Anber-Sorbo inflation}

In some theories, the constraint in eq. (3.1) will be a mild constraint. In others, it is a much more difficult constraint to satisfy. An example of the latter case is chromonatural inflation. In this section, we will go through a clockworkable example: the Anber-Sorbo inflation model [4].

The Anber-Sorbo model is based on natural inflation with an axion-like particle being the inflaton [86]. The usual natural inflation model needs the axion field range to be super-Planckian to satisfy the slow-roll condition. What is new in the Anber-Sorbo model is that a large axion-gauge field coupling leads to production of gauge bosons when the axion rolls down the potential. The electromagnetic dissipation slows down the rolling and relaxes the slow-roll constraint. As a result, the field range of the axion inflaton could be sub-Planckian. Below we review the basics of the model and its predictions briefly.

The Lagrangian of the model is

$$
\mathcal{L}=\frac{1}{2}(\partial a)^{2}+\sum_{i=1}^{\mathcal{N}} \frac{1}{4} F_{\mu \nu}^{i} F^{\mu \nu ; i}+\mu^{4} \cos \left(\frac{a}{f_{a}}\right)+\sum_{i=1}^{\mathcal{N}} \frac{k j \alpha}{8 \pi} \frac{a}{f_{a}} F_{\mu \nu}^{i} \widetilde{F}^{\mu \nu ; i},
$$


where the potential and interaction terms are the same as in eq. (1.1) by expressing $F_{a}=$ $f_{a} / j$. The index $i=1,2, \cdots \mathcal{N}$ labels the $\mathcal{N} \mathrm{U}(1)$ gauge fields introduced in the model. The large number of gauge fields is necessary to get the right amplitude of the two-point function, as we discuss below. Again $\alpha=e^{2} /(4 \pi)$ here is the fine structure constant of the gauged U(1)'s (note that in the original paper, $\alpha$ is used as the overall coupling equivalent to $k j \alpha /(2 \pi)$ here). We assume that the gauge couplings of all the $\mathrm{U}(1)$ 's are the same without loss of generality. We also assume the couplings of axion to all the gauge fields are the same as well. The key parameter that controls the tachyonic production of gauge fields is $\xi \equiv \beta \frac{\dot{a}}{2 f_{a} H}$. In the slow-roll solution, it satisfies

$$
\xi \approx \frac{2}{\pi} \log \left(\xi \frac{14 M_{\mathrm{pl}}}{(\mathcal{N} \beta)^{1 / 4} \mu}\right), \quad \text { with } \quad \beta=\frac{k j \alpha}{2 \pi}
$$

where $\mathcal{O}(1) \lesssim \xi \lesssim 20$ and the reduced Planck scale $M_{\mathrm{pl}}=2.4 \times 10^{18} \mathrm{GeV}$. The total number of e-folds is given by

$$
N_{e}=\frac{\beta}{2 \xi} \frac{\Delta a}{f_{a}} \lesssim \frac{\pi \beta}{2 \xi}
$$

where $\Delta a$ is the range of variation of $a$ during inflation, which is bounded to be $\lesssim \pi f_{a}$.

The amplitude of the two-point power spectrum is given by [14]

$$
\Delta_{\mathcal{R}}^{2} \approx \frac{0.05}{\mathcal{N} \xi^{2}}
$$

and should match the observation $2.2 \times 10^{-9}$ [87]. Since $\xi$ is at most around 20, $\mathcal{N}>5.6 \times 10^{4}$. In this model, the generation of photons during inflation sources chiral gravitational waves. As a result, it adds an additional contribution to the tensor to scalar ratio on top of the usual quantum fluctuations of gravitons [14]

$$
\begin{aligned}
r & =\frac{1}{P_{\xi}} \frac{2 V}{3 \pi^{2} M_{\mathrm{pl}}^{4}}+2.7 \times 10^{2} \frac{\xi^{4}}{\beta^{2}}\left(\frac{V^{\prime} f_{a}}{V}\right)^{2}, \\
& =5.2 \times 10^{4} \frac{\xi^{6}}{\beta} \mathrm{e}^{-2 \pi \xi}+2.7 \times 10^{2} \frac{\xi^{4}}{\beta^{2}},
\end{aligned}
$$

where to get the second line, we approximate $V \approx \mu^{4}, V^{\prime} f \approx V$ and express $\mu$ in terms of $\xi$ and $\beta$ using eq. (4.2).

To get enough e-folds with $N_{e} \approx(50-60)$ and to satisfy the observational constraint $r \lesssim 0.11$ [87], we need a large enhancement of all the axion-photon couplings:

$$
\begin{aligned}
& \text { When } \xi=10, \quad \beta \gtrsim 5 \times 10^{3} \Rightarrow k j \gtrsim 5 \times 10^{4}\left(\frac{0.1}{\alpha}\right) \\
& \text { When } \xi=20, \quad \beta \gtrsim 2 \times 10^{4} \Rightarrow k j \gtrsim 2 \times 10^{5}\left(\frac{0.1}{\alpha}\right)
\end{aligned}
$$

To obtain this large enhancement, we need to invoke the clockwork mechanism as described in section 3.3. For instance, it could be realized in the confinement tower model with 10 
confining $\mathrm{SU}(3)$ 's and 10 axion-like particles (the lightest axion being the inflaton) when $\xi=10$. In this case, the fundamental period of the axion inflaton is

$$
F_{a}=\frac{f_{a}}{j}=2 \times 10^{-5} f_{a}\left(\frac{\alpha}{0.1}\right),
$$

where we take $k=1$ without loss of generality. The confinement scale $\mu$ is given by,

$$
\mu=14 M_{\mathrm{pl}} \frac{\xi}{(\mathcal{N} \beta)^{1 / 4}} \mathrm{e}^{-\pi \xi / 2} .
$$

Since it is exponentially sensitive to the order one parameter $\xi$, the constraint in eq. (3.1) and sub-Planckian field range $f_{a}<M_{\mathrm{pl}}$ could be satisfied simultaneously, for instance, when $\xi=10, \alpha=0.1, \beta=5 \times 10^{3}$ and $f_{a}=10^{18} \mathrm{GeV}, \mu=2.8 \times 10^{11} \mathrm{GeV}$ and $F_{a}=2 \times 10^{13} \mathrm{GeV}$.

In short, the Anber-Sorbo model needs two types of large numbers: a) $\mathcal{O}\left(10^{5}\right) \mathrm{U}(1)$ gauge fields; $b$ ) each axion-gauge field coupling enhanced by at least $\mathcal{O}\left(10^{5}\right)$ which could result from clockworking of $\mathcal{O}(10)$ axions. In addition to predicting chiral gravitational waves, Anber-Sorbo model also predicts a non-Gaussianity $f_{\mathrm{NL}}^{\text {equil }}=-1.3 \xi$, which is in the range (5-30). ${ }^{3}$ This is consistent with current Planck constraint [90] and could be confirmed or falsified at CMB stage 4 experiment. Finally, refs. [6, 91] also consider the possibility that the large number of gauge fields produced during inflation lead to thermalization and formation of a hot plasma. We stick to the original proposal of Anber and Sorbo and leave the possibility of thermalization for future discussion.

\section{Chromonatural inflation}

Chromonatural inflation models feature an axion with a large coupling to gauge fields, requiring a very large enhancement factor $j k$ - much larger than the $\mathcal{O}(100)$ number usually stated in the literature. The basic ingredients are an axion which has a potential and a coupling to a non-Abelian gauge field:

$$
\mathcal{L}=-\frac{1}{4} F_{\mu \nu}^{a} F^{a, \mu \nu}+\mu^{4} \cos \left(\frac{a}{f_{a}}\right)+\frac{k j \alpha}{8 \pi f_{a}} a F_{\mu \nu}^{a} \widetilde{F}^{a, \mu \nu}
$$

For this simple analysis, we ignore the fact that we need to add a Higgs for consistency with inflationary phenomenology [92]. We expect this to make order-one differences in allowed parameters, but not to qualitatively change any conclusion. ${ }^{4}$ The original literature on chromonatural inflation introduced a parameter $\lambda \equiv k j \alpha / \pi$ and argued that a modestly large $\lambda \sim 100$ was necessary for the mechanism to work. However, their benchmark model invoked a tiny gauge coupling $\alpha \sim 10^{-12}$, and hence (implicitly) a huge $k j \sim 10^{15}$. (This

\footnotetext{
${ }^{3}$ Possible large non-Gaussianity in inflation models with axion coupling to gauge fields have been studied in refs. $[88,89]$. Yet those analyses assume the back-reaction of the gauge field on the inflaton is negligible and inflation potential is flat. Thus their derived constraint on the axion-gauge field coupling does not apply to the Anber-Sorbo model.

${ }^{4}$ An alternative, more qualitatively different, approach is to introduce a separate inflaton field $\phi$, treating both the axion and the gauge field as spectators during inflation; see e.g. [18, 93, 94]. The constraints we discuss should play a role in those models as well, but we will not consider them in detail here.
} 
has previously been pointed out, in passing, in [95].) We will now show that the clockwork mechanism is not sufficient to explain this large number.

The gauge field is assumed to have a background,

$$
F_{0 i}^{a}=\partial_{t}(\psi(t) \mathrm{a}(t)) \delta_{i}^{a}, \quad F_{i j}^{a}=-g f_{i j}^{a}(\psi(t) \mathrm{a}(t))^{2}
$$

where $\mathrm{a}(t)$ refers to the scale factor. This classical background spontaneously breaks the product of spatial rotations and internal gauge rotations to the diagonal, preserving a notion of isotropy. Such inflationary backgrounds were first explored in the context of gaugeflation [96], which resembles chromonatural inflation [97] but relies on higher dimension operators instead of an axion field (and so is outside the scope of our discussion here). We are interested in solutions where the energy density is dominated by the axion $a$, and it has a slow roll solution supported by friction from the gauge field. A useful quantity to parametrize the solutions is

$$
w \equiv\left(\frac{k j \mu^{4}}{6 \pi^{2} M_{\mathrm{pl}}^{4}}\right)^{1 / 3}
$$

(This is approximately $1 / m_{\psi}$ in the notation of $[11,15]$.) It turns out that for $w>1$ fluctuations are unstable $[11,98]$ which will drastically change the phenomenology, so we restrict to $w \lesssim 1$. The equations of motion imply that (approximately),

$$
\begin{aligned}
\frac{\psi}{M_{\mathrm{pl}}} & \approx \frac{\mu^{2}}{g w M_{\mathrm{pl}}^{2}} \sqrt{2 / 3} \sin ^{1 / 3} \theta \\
\frac{1}{f_{a}} \frac{d a}{d N} & =\frac{2 \pi}{k j \alpha}\left(\frac{w \cos \theta}{\sin ^{1 / 3} \theta}+\frac{\sin ^{1 / 3} \theta}{w \cos \theta}\right)
\end{aligned}
$$

with $\theta \equiv a /\left(2 f_{a}\right)$.

It follows that the number of e-folds in chromonatural inflation is given by

$$
N_{e}\left(a_{0}\right)=\frac{k j \alpha}{\pi} \int_{a_{0} / 2 f_{a}}^{\pi / 2} d \theta \frac{1}{\frac{w \cos \theta}{\sin ^{1 / 3} \theta}+\frac{\sin ^{1 / 3} \theta}{w \cos \theta}} \lesssim \frac{3}{2 \pi} \alpha k j w
$$

where we have used the fact that $w \lesssim 1$ to derive the final inequality. Our constraint eq. (3.1) implies that $j \mu<f_{a}$, so that

$$
\alpha k j w=\alpha\left(\frac{k^{4} j^{4} \mu^{4}}{6 \pi^{2} M_{\mathrm{pl}}^{4}}\right)^{1 / 3}<\alpha k\left(\frac{k f_{a}^{4}}{6 \pi^{2} M_{\mathrm{pl}}^{4}}\right)^{1 / 3} \lesssim\left(\frac{k}{6 \pi^{2}}\right)^{1 / 3},
$$

where in the last step we have assumed a sub-Planckian field range $f_{a} \lesssim M_{\mathrm{pl}}$ as well as the perturbativity bound $\alpha k \lesssim 1$.

Numerically, this requires that $k$ be quite large:

$$
k \gtrsim 6 \pi^{2}\left(\frac{2 \pi N_{e}}{3}\right)^{3} \sim 10^{8}\left(N_{e} / 60\right)^{3} .
$$


Because the clockwork mechanism can explain a large $j$ but not a large $k$, this immediately implies that clockwork alone is not sufficient for a viable chromonatural inflation cosmology (if we restrict to sub-Planckian field ranges). At the same time, the constraint $k \alpha \lesssim 1$ implies that a large value of $k$ is also not sufficient by itself: we would never attain $\lambda \gtrsim 1$ by relying solely on $k$. Therefore, we conclude that we need both a clockwork mechanism as well as (very) large charges in order to realize chromonatural inflation. The large value of $k$ could potentially arise from kinetic mixing with another, lighter axion, but in this case one should check whether the additional light field has any important phenomenological consequences. We will not consider this possibility in more detail here.

Inflationary phenomenology further restricts the allowed parameter space significantly. The other constraints on this parameter space come from the size of the scalar perturbations, the spectral tilt, and the slow-roll parameter. In the case of chromonatural inflation, these have been calculated in some detail $[9,11,15,98,99]$. The scaling behavior of these quantities is [11]

$$
\begin{aligned}
\epsilon_{H} & \sim \eta_{H} \sim 1-n_{s} \sim \frac{1}{N_{e}} \sim \frac{2 \pi}{3 k j \alpha w} \\
\Delta_{\mathcal{R}}^{2} & \sim \frac{\mu^{4}}{6 \epsilon_{H} M_{\mathrm{pl}}{ }^{4}} \sim \frac{\mu^{4}}{6 M_{\mathrm{pl}}{ }^{4}} \frac{3}{2 \pi} k j \alpha w .
\end{aligned}
$$

We emphasize that these relations can get corrected by $\mathcal{O}(1)$ factors, especially once we consider the Higgsed chromonatural model [92], but the overall scaling behavior should still hold.

From these equations, we can immediately extract that the value of $\mu$ must be large:

$$
\mu \sim M_{\mathrm{pl}}\left(\frac{\Delta_{\mathcal{R}}^{2}}{N_{e}}\right)^{1 / 4} \sim 10^{16} \mathrm{GeV}
$$

As discussed in [95], this may superficially run afoul of the Weak Gravity Conjecture, which motivates an ultraviolet cutoff at or below $g M_{\mathrm{pl}}$ [28]. Nonetheless, this UV cutoff may be of a mild form, associated with the existence of a tower of states charged under the gauge theory [100-103]. This leads to a more stringent UV cutoff at or below $g^{1 / 2} M_{\mathrm{pl}}$, with which chromonatural inflation is at best marginally compatible [95]. However, in the context of clockwork completions (5.11) has an immediate, less conjectural, consequence. The fundamental clockwork constraint (3.1) tells us that $\mu \lesssim f_{a} / j \lesssim M_{\mathrm{pl}} / j$ (where we again assume a sub-Planckian field range), and hence

$$
j \lesssim \frac{M_{\mathrm{pl}}}{\mu} \sim 10^{2}
$$

This implies that (in the notation of the original references) the benchmark values of $\lambda$ are only marginally compatible with the clockwork mechanism, and compatibility with this bound further requires us to choose $k \sim \alpha^{-1}$ so that the theory is at or near strong coupling. Notice, in particular, that our arguments require that $k$ is several orders of magnitude larger than $j$, so that clockwork alone falls dramatically short of explaining the large coupling. 




Figure 1. Viable parameter space for chromonatural inflation. We assume the number of e-folds of inflation, $N_{e}=60$, and $\Delta_{\mathcal{R}}^{2}=2.2 \times 10^{-9}$ as well as other theoretically motivated constraints discussed in the text. The red regions show the allowed parameter space for particular values of $k$. The white region is the union of all such regions. The fact that large $k$ is required indicates that clockwork alone cannot explain the large coupling in chromonatural inflation.

The two inflationary constraints above can be used to eliminate $\mu$ and $j$, leaving $\left\{\alpha, F_{a}, k\right\}$ as the free parameters. ${ }^{5}$ There are a number of inequalities that these parameters should satisfy,

- $k \alpha<1$ : from perturbativity of the gauge coupling

- $w<1$ : for stability of perturbations

- $\mu \lesssim F_{a}$ : as derived in equation (3.1)

- $f_{a}=F_{a} j<M_{\mathrm{pl}}$ : avoiding large field excursions is one of the prime motivations of the model

These constraints are shown in figure 1. (A further constraint, that $j>1$, is satisfied everywhere in the figure.) For a particular choice of $k$, the red triangles show the regions allowed by the constraints above. The upper edge of the triangle corresponds to $k \alpha=1$, whereas the sloping edge corresponds to $f_{a}=M_{\mathrm{pl}}$. The white region is the union of all such regions. We see that the allowed range of parameters is roughly $\alpha \in\left(10^{-10}, 10^{-8}\right)$, $\frac{F_{a}}{M_{\mathrm{pl}}} \in(0.004,0.008), j \in(130,260)$ and $k \in\left(10^{7}, 10^{9}\right)$.

\footnotetext{
${ }^{5}$ Because we expect that a model compatible with data in detail will involve various changes to order one factors, as in [92], we have simply taken the $\sim$ estimates in (5.10) to be equalities and have fixed the number of e-folds of inflation $N_{e}=60$. Our goal is to convey an order-of-magnitude sense of the constraints, and the precise order-one factors appearing in our results should not be taken too seriously.
} 


\section{Conclusions}

Large couplings of axion to gauge fields could lead to very interesting cosmology. In particular, they may allow alternative realizations of slow-roll inflation with a steep inflaton potential, as suggested by Anber-Sorbo and chromonatural inflation models. In this article, we demonstrate that quite a few axion cosmology models, including Anber-Sorbo and chromonatural models, actually require (significantly) larger axion-gauge field couplings than quoted in the literature, taking into proper account of the presence of the gauge coupling strength in the axion coupling when gauge fields are canonically normalized. This observation imposes a highly non-trivial model-building challenge to axion cosmology: can a large axion coupling to gauge fields arise in a UV completion without large input parameters?

We focus on the two simplest possible methods: inclusion of matter with large charges under the gauge group or PQ symmetry and the clockwork mechanism (equivalently, multiaxion alignment) to significantly extend the effective axion field range beyond its fundamental period. We discuss simple theoretical constraints in either case: in the large charge case, the strong coupling constraint requires the charge times the gauge coupling strength to be around or below one while in the clockwork case, the confinement scale of the nonperturbative dynamics generating the axion potential has to be below the fundamental period of the axion field. These constraints obstruct the UV completion, point towards different feasible parameter space, and may alter the phenomenological predictions.

Finally, we want to comment on possible future directions. In our study, we only use simple parametric approximations for some of the observables. It could be worthwhile to perform a more systematic analysis of the cosmological observables in the possible UV completions. Another possible approach to enhance the axion coupling, the kinetic mixing route, is largely unexplored in our paper. It will be interesting to pursue it further and study the dynamics of the additional lighter axion that is needed in this approach.

\section{Acknowledgments}

We thank Patrick Draper, David Pinner, and Jure Zupan for discussions. PA would like to thank the Kavli Institute for Theoretical Physics for their hospitality during the completion of this work. PA is supported by the NSF grants PHY-0855591 and PHY-1216270. JF is supported by the DOE grant DE-SC-0010010. MR is supported in part by the DOE grant DE-SC0013607 and the NASA grant NNX16AI12G.

\section{A How confining models ensure $\mu<f_{a}$}

We have argued that any effective potential containing the cosine potential (3.2) requires $\mu \lesssim f_{a}$. Here we elaborate on this point by showing how the inequality is enforced in some simple examples. If we try to write down a model that will produce $\mu \gtrsim f_{a}$, we will find that confinement breaks the PQ symmetry and $f_{a}$ is larger than expected, consistent with this bound. For example, consider the theory where our axion arises from the renormalizable 
Lagrangian

$$
-\lambda\left(|\phi|^{2}-\frac{1}{2} v_{\mathrm{PQ}}^{2}\right)^{2}+(y \phi Q \widetilde{Q}+\text { h.c. }),
$$

with $Q, \widetilde{Q}$ fermions charged in the fundamental and antifundamental representations of an $\mathrm{SU}(N)$ gauge theory. At first glance, the axion decay constant $f_{a}$ is set by $v_{\mathrm{PQ}}$ whereas the scale $\mu$ that will appear in front of the cosine potential is determined by confinement, so we can tune the two parameters independently. However, despite this freedom we cannot attain $\mu \gg f_{a}$. Confinement produces a quark condensate $\langle Q \widetilde{Q}\rangle \equiv v_{\text {conf }}^{3}$, which is itself a breaking of the PQ symmetry. In turn, this produces an effective tadpole for the scalar field $\phi$, potentially changing its vev. Ignoring radial fluctuations, we can work in terms of two effective pseudoscalar fields $a$ and $\eta^{\prime}$ :

$$
\phi \mapsto \frac{1}{\sqrt{2}} f_{a} \mathrm{e}^{\mathrm{i} a(x) / f_{a}} \quad \text { and } Q \widetilde{Q}=v_{\text {conf }}^{3} \mathrm{e}^{\mathrm{i} \eta^{\prime}(x) / f_{\eta^{\prime}}} .
$$

After confinement the effective potential for these fields is

$$
V\left(a, \eta^{\prime}\right) \approx \sqrt{2} y v_{\mathrm{conf}}^{3} f_{a} \cos \left(\frac{a}{f_{a}}+\frac{\eta^{\prime}}{f_{\eta^{\prime}}}\right)+\Lambda_{\mathrm{conf}}^{4} \cos \left(\frac{\eta^{\prime}}{f_{\eta^{\prime}}}\right) .
$$

First consider the limit where the tadpole term makes little difference in the vev of $\phi$, i.e. when $f_{a} \approx v_{\mathrm{PQ}}$. The condition for this to hold is that $y v_{\text {conf }}^{3} \ll \lambda f^{3} \lesssim f^{3}$. In this case, we have $\mu^{4} \sim y v_{\text {conf }}^{3} f_{a} \ll f_{a}^{4}$, in line with the bound above. On the other hand, it could be that the tadpole term is very significant and that $f_{a} \gg v_{\mathrm{PQ}}$. In this case the vev of $\phi$ is determined primarily by the interplay between the quartic and tadpole terms, and hence

$$
f_{a} \sim\left(\frac{y}{\lambda}\right)^{1 / 3} v_{\text {conf }}
$$

If $\lambda$ is small then this can still be the dominant PQ-breaking vev, but the effective potential for the axion then scales as $y v_{\text {conf }}^{3} f_{a} \sim \lambda f_{a}^{4} \lesssim f_{a}^{4}$, again in accord with the general bound.

Alternatively, we could consider a theory where the field $\phi$ dominantly has a PQstabilizing mass term, i.e. the Lagrangian is

$$
-m^{2}|\phi|^{2}+(y \phi Q \widetilde{Q}+\text { h.c. }) \text {, }
$$

and so PQ-breaking is entirely driven by confinement. In that case one has

$$
f_{a} \sim \frac{y v_{\mathrm{conf}}^{3}}{m^{2}} .
$$

To achieve $f_{a} \ll \mu$ we could attempt to take $m \gg v_{\text {conf }}$. However, in that limit, we find that the $\eta^{\prime}$ becomes lighter than the axion, which has a mass

$$
m_{a}^{2} \sim m^{2}
$$

As a result, we should integrate out the axion and view $\eta^{\prime}$ as the dynamical field, rather than the alternative.

In this way, we see that concrete models of Peccei-Quinn breaking beginning from renormalizable theories will always respect the perturbative unitarity bounds that we have discussed above. 
Open Access. This article is distributed under the terms of the Creative Commons Attribution License (CC-BY 4.0), which permits any use, distribution and reproduction in any medium, provided the original author(s) and source are credited.

\section{References}

[1] M.S. Turner and L.M. Widrow, Inflation Produced, Large Scale Magnetic Fields, Phys. Rev. D 37 (1988) 2743 [INSPIRE].

[2] W.D. Garretson, G.B. Field and S.M. Carroll, Primordial magnetic fields from pseudoGoldstone bosons, Phys. Rev. D 46 (1992) 5346 [hep-ph/9209238] [INSPIRE].

[3] P. Adshead, J.T. Giblin, T.R. Scully and E.I. Sfakianakis, Magnetogenesis from axion inflation, JCAP 10 (2016) 039 [arXiv:1606.08474] [INSPIRE].

[4] M.M. Anber and L. Sorbo, Naturally inflating on steep potentials through electromagnetic dissipation, Phys. Rev. D 81 (2010) 043534 [arXiv:0908.4089] [InSPIRE].

[5] A. Notari and K. Tywoniuk, Dissipative Axial Inflation, JCAP 12 (2016) 038 [arXiv: 1608.06223] [INSPIRE].

[6] R.Z. Ferreira and A. Notari, Thermalized Axion Inflation, JCAP 09 (2017) 007 [arXiv: 1706.00373] [INSPIRE].

[7] S. H.-S. Alexander, M.E. Peskin and M.M. Sheikh-Jabbari, Leptogenesis from gravity waves in models of inflation, Phys. Rev. Lett. 96 (2006) 081301 [hep-th/0403069] [INSPIRE].

[8] P. Adshead and M. Wyman, Chromo-Natural Inflation: Natural inflation on a steep potential with classical non-Abelian gauge fields, Phys. Rev. Lett. 108 (2012) 261302 [arXiv:1202.2366] [INSPIRE].

[9] E. Dimastrogiovanni, M. Fasiello and A.J. Tolley, Low-Energy Effective Field Theory for Chromo-Natural Inflation, JCAP 02 (2013) 046 [arXiv: 1211.1396] [INSPIRE].

[10] A. Maleknejad, M.M. Sheikh-Jabbari and J. Soda, Gauge Fields and Inflation, Phys. Rept. 528 (2013) 161 [arXiv:1212.2921] [INSPIRE].

[11] P. Adshead, E. Martinec and M. Wyman, Perturbations in Chromo-Natural Inflation, JHEP 09 (2013) 087 [arXiv:1305.2930] [InSPIRE].

[12] A. Lue, L.-M. Wang and M. Kamionkowski, Cosmological signature of new parity violating interactions, Phys. Rev. Lett. 83 (1999) 1506 [astro-ph/9812088] [INSPIRE].

[13] S. Alexander and J. Martin, Birefringent gravitational waves and the consistency check of inflation, Phys. Rev. D 71 (2005) 063526 [hep-th/0410230] [INSPIRE].

[14] M.M. Anber and L. Sorbo, Non-Gaussianities and chiral gravitational waves in natural steep inflation, Phys. Rev. D 85 (2012) 123537 [arXiv:1203.5849] [INSPIRE].

[15] P. Adshead, E. Martinec and M. Wyman, Gauge fields and inflation: Chiral gravitational waves, fluctuations and the Lyth bound, Phys. Rev. D 88 (2013) 021302 [arXiv:1301.2598] [INSPIRE].

[16] A. Maleknejad, Axion Inflation with an $\mathrm{SU}(2)$ Gauge Field: Detectable Chiral Gravity Waves, JHEP 07 (2016) 104 [arXiv: 1604.03327] [INSPIRE].

[17] I. Obata and J. Soda, Oscillating Chiral Tensor Spectrum from Axionic Inflation, Phys. Rev. D 94 (2016) 044062 [arXiv: 1607.01847] [INSPIRE]. 
[18] E. Dimastrogiovanni, M. Fasiello and T. Fujita, Primordial Gravitational Waves from Axion-Gauge Fields Dynamics, JCAP 01 (2017) 019 [arXiv: 1608.04216] [INSPIRE].

[19] P. Adshead, J.T. Giblin, T.R. Scully and E.I. Sfakianakis, Gauge-preheating and the end of axion inflation, JCAP 12 (2015) 034 [arXiv: 1502.06506] [INSPIRE].

[20] P. Agrawal, G. Marques-Tavares and W. Xue, Opening up the QCD axion window, JHEP 03 (2018) 049 [arXiv: 1708.05008] [INSPIRE].

[21] N. Kitajima, T. Sekiguchi and F. Takahashi, Cosmological abundance of the QCD axion coupled to hidden photons, Phys. Lett. B 781 (2018) 684 [arXiv:1711.06590] [inSPIRE].

[22] A. Hook and G. Marques-Tavares, Relaxation from particle production, JHEP 12 (2016) 101 [arXiv: 1607.01786] [INSPIRE].

[23] W. Tangarife, K. Tobioka, L. Ubaldi and T. Volansky, Relaxed Inflation, arXiv: 1706.00438 [INSPIRE].

[24] W. Tangarife, K. Tobioka, L. Ubaldi and T. Volansky, Dynamics of Relaxed Inflation, JHEP 02 (2018) 084 [arXiv: 1706.03072] [INSPIRE].

[25] P.W. Graham, D.E. Kaplan and S. Rajendran, Cosmological Relaxation of the Electroweak Scale, Phys. Rev. Lett. 115 (2015) 221801 [arXiv:1504.07551] [INSPIRE].

[26] D.H. Lyth, What would we learn by detecting a gravitational wave signal in the cosmic microwave background anisotropy?, Phys. Rev. Lett. 78 (1997) 1861 [hep-ph/9606387] [INSPIRE].

[27] C. Vafa, The String landscape and the swampland, hep-th/0509212 [INSPIRE].

[28] N. Arkani-Hamed, L. Motl, A. Nicolis and C. Vafa, The String landscape, black holes and gravity as the weakest force, JHEP 06 (2007) 060 [hep-th/0601001] [INSPIRE].

[29] H. Ooguri and C. Vafa, On the Geometry of the String Landscape and the Swampland, Nucl. Phys. B 766 (2007) 21 [hep-th/0605264] [inSPIRE].

[30] T. Banks, M. Dine, P.J. Fox and E. Gorbatov, On the possibility of large axion decay constants, JCAP 06 (2003) 001 [hep-th/0303252] [INSPIRE].

[31] T. Rudelius, On the Possibility of Large Axion Moduli Spaces, JCAP 04 (2015) 049 [arXiv: 1409.5793] [INSPIRE].

[32] J.P. Conlon and S. Krippendorf, Axion decay constants away from the lamppost, JHEP 04 (2016) 085 [arXiv: 1601.00647] [InSPIRE].

[33] F. Baume and E. Palti, Backreacted Axion Field Ranges in String Theory, JHEP 08 (2016) 043 [arXiv: 1602.06517] [INSPIRE].

[34] L. McAllister, P. Schwaller, G. Servant, J. Stout and A. Westphal, Runaway Relaxion Monodromy, JHEP 02 (2018) 124 [arXiv:1610.05320] [INSPIRE].

[35] M. Cicoli, D. Ciupke, C. Mayrhofer and P. Shukla, A Geometrical Upper Bound on the Inflaton Range, JHEP 05 (2018) 001 [arXiv: 1801.05434] [INSPIRE].

[36] R. Blumenhagen, D. Kläwer, L. Schlechter and F. Wolf, The Refined Swampland Distance Conjecture in Calabi-Yau Moduli Spaces, JHEP 06 (2018) 052 [arXiv:1803.04989] [INSPIRE].

[37] T. Rudelius, Constraints on Axion Inflation from the Weak Gravity Conjecture, JCAP 09 (2015) 020 [arXiv: 1503.00795] [InSPIRE]. 
[38] M. Montero, A.M. Uranga and I. Valenzuela, Transplanckian axions!?, JHEP 08 (2015) 032 [arXiv: 1503.03886] [INSPIRE].

[39] J. Brown, W. Cottrell, G. Shiu and P. Soler, Fencing in the Swampland: Quantum Gravity Constraints on Large Field Inflation, JHEP 10 (2015) 023 [arXiv: 1503.04783] [INSPIRE].

[40] T.C. Bachlechner, C. Long and L. McAllister, Planckian Axions and the Weak Gravity Conjecture, JHEP 01 (2016) 091 [arXiv: 1503.07853] [INSPIRE].

[41] A. Hebecker, P. Mangat, F. Rompineve and L.T. Witkowski, Winding out of the Swamp: Evading the Weak Gravity Conjecture with F-term Winding Inflation?, Phys. Lett. B 748 (2015) 455 [arXiv: 1503.07912] [INSPIRE].

[42] J. Brown, W. Cottrell, G. Shiu and P. Soler, On Axionic Field Ranges, Loopholes and the Weak Gravity Conjecture, JHEP 04 (2016) 017 [arXiv: 1504.00659] [INSPIRE].

[43] B. Heidenreich, M. Reece and T. Rudelius, Weak Gravity Strongly Constrains Large-Field Axion Inflation, JHEP 12 (2015) 108 [arXiv: 1506.03447] [INSPIRE].

[44] A. Nicolis, On Super-Planckian Fields at Sub-Planckian Energies, JHEP 07 (2008) 023 [arXiv: 0802.3923] [INSPIRE].

[45] D. Klaewer and E. Palti, Super-Planckian Spatial Field Variations and Quantum Gravity, JHEP 01 (2017) 088 [arXiv:1610.00010] [INSPIRE].

[46] I. Valenzuela, Backreaction Issues in Axion Monodromy and Minkowski 4-forms, JHEP 06 (2017) 098 [arXiv: 1611.00394] [INSPIRE].

[47] M.J. Dolan, P. Draper, J. Kozaczuk and H. Patel, Transplanckian Censorship and Global Cosmic Strings, JHEP 04 (2017) 133 [arXiv: 1701.05572] [INSPIRE].

[48] R. Blumenhagen, I. Valenzuela and F. Wolf, The Swampland Conjecture and F-term Axion Monodromy Inflation, JHEP 07 (2017) 145 [arXiv: 1703.05776] [INSPIRE].

[49] T.W. Grimm, E. Palti and I. Valenzuela, Infinite Distances in Field Space and Massless Towers of States, JHEP 08 (2018) 143 [arXiv: 1802.08264] [INSPIRE].

[50] B. Heidenreich, M. Reece and T. Rudelius, Emergence of Weak Coupling at Large Distance in Quantum Gravity, Phys. Rev. Lett. 121 (2018) 051601 [arXiv:1802.08698] [INSPIRE].

[51] A. Landete and G. Shiu, Mass Hierarchies and Dynamical Field Range, Phys. Rev. D 98 (2018) 066012 [arXiv: 1806.01874] [INSPIRE].

[52] T.D. Brennan, F. Carta and C. Vafa, The String Landscape, the Swampland and the Missing Corner, PoS (TASI2017) 015 (2017) [arXiv: 1711.00864] [INSPIRE].

[53] S. Sethi, Supersymmetry Breaking by Fluxes, JHEP 10 (2018) 022 [arXiv:1709.03554] [INSPIRE].

[54] U.H. Danielsson and T. Van Riet, What if string theory has no de Sitter vacua?, Int. J. Mod. Phys. D 27 (2018) 1830007 [arXiv:1804.01120] [INSPIRE].

[55] J. Moritz and T. Van Riet, Racing through the swampland: de Sitter uplift vs weak gravity, JHEP 09 (2018) 099 [arXiv: 1805. 00944] [INSPIRE].

[56] A. Rajaraman, de Sitter Space is Unstable in Quantum Gravity, Phys. Rev. D 94 (2016) 125025 [arXiv: 1608.07237 ] [INSPIRE].

[57] D. Kutasov, T. Maxfield, I. Melnikov and S. Sethi, Constraining de Sitter Space in String Theory, Phys. Rev. Lett. 115 (2015) 071305 [arXiv: 1504.00056] [InSPIRE]. 
[58] J. Moritz, A. Retolaza and A. Westphal, Toward de Sitter space from ten dimensions, Phys. Rev. D 97 (2018) 046010 [arXiv: 1707.08678] [INSPIRE].

[59] G. Obied, H. Ooguri, L. Spodyneiko and C. Vafa, de Sitter Space and the Swampland, arXiv: 1806.08362 [INSPIRE].

[60] P. Agrawal, G. Obied, P.J. Steinhardt and C. Vafa, On the Cosmological Implications of the String Swampland, Phys. Lett. B 784 (2018) 271 [arXiv:1806.09718] [INSPIRE].

[61] E. Witten, Large N Chiral Dynamics, Annals Phys. 128 (1980) 363 [InSPIRE].

[62] E. Witten, Theta dependence in the large $N$ limit of four-dimensional gauge theories, Phys. Rev. Lett. 81 (1998) 2862 [hep-th/9807109] [INSPIRE].

[63] E. Silverstein and A. Westphal, Monodromy in the CMB: Gravity Waves and String Inflation, Phys. Rev. D 78 (2008) 106003 [arXiv:0803.3085] [INSPIRE].

[64] M. Farina, D. Pappadopulo, F. Rompineve and A. Tesi, The photo-philic QCD axion, JHEP 01 (2017) 095 [arXiv: 1611.09855] [INSPIRE].

[65] P. Agrawal, J. Fan, M. Reece and L.-T. Wang, Experimental Targets for Photon Couplings of the QCD Axion, JHEP 02 (2018) 006 [arXiv:1709.06085] [INSPIRE].

[66] K.S. Babu, S.M. Barr and D. Seckel, Axion dissipation through the mixing of Goldstone bosons, Phys. Lett. B 336 (1994) 213 [hep-ph/9406308] [INSPIRE].

[67] J.E. Kim, H.P. Nilles and M. Peloso, Completing natural inflation, JCAP 01 (2005) 005 [hep-ph/0409138] [INSPIRE].

[68] K. Choi and S.H. Im, Realizing the relaxion from multiple axions and its UV completion with high scale supersymmetry, JHEP 01 (2016) 149 [arXiv:1511.00132] [INSPIRE].

[69] D.E. Kaplan and R. Rattazzi, Large field excursions and approximate discrete symmetries from a clockwork axion, Phys. Rev. D 93 (2016) 085007 [arXiv:1511.01827] [INSPIRE].

[70] T. Kobayashi and N. Afshordi, Schwinger Effect in $4 D$ de Sitter Space and Constraints on Magnetogenesis in the Early Universe, JHEP 10 (2014) 166 [arXiv:1408.4141] [INSPIRE].

[71] T. Hayashinaka, T. Fujita and J. Yokoyama, Fermionic Schwinger effect and induced current in de Sitter space, JCAP 07 (2016) 010 [arXiv: 1603.04165] [INSPIRE].

[72] T. Hayashinaka and J. Yokoyama, Point splitting renormalization of Schwinger induced current in de Sitter spacetime, JCAP 07 (2016) 012 [arXiv:1603.06172] [INSPIRE].

[73] T. Hayashinaka and S.-S. Xue, Physical renormalization condition for de Sitter QED, Phys. Rev. D 97 (2018) 105010 [arXiv: 1802.03686] [INSPIRE].

[74] K.D. Lozanov, A. Maleknejad and E. Komatsu, Schwinger Effect by an SU(2) Gauge Field during Inflation, arXiv:1805.09318 [INSPIRE].

[75] A.J. Long, Cosmological Aspects of the Clockwork Axion, JHEP 07 (2018) 066 [arXiv: 1803.07086] [INSPIRE].

[76] J.E. Kim, Weak Interaction Singlet and Strong CP Invariance, Phys. Rev. Lett. 43 (1979) 103 [INSPIRE].

[77] M.A. Shifman, A.I. Vainshtein and V.I. Zakharov, Can Confinement Ensure Natural CP Invariance of Strong Interactions?, Nucl. Phys. B 166 (1980) 493 [INSPIRE].

[78] T.C. Bachlechner, M. Dias, J. Frazer and L. McAllister, Chaotic inflation with kinetic alignment of axion fields, Phys. Rev. D 91 (2015) 023520 [arXiv:1404.7496] [INSPIRE]. 
[79] G. Shiu, W. Staessens and F. Ye, Widening the Axion Window via Kinetic and Stückelberg Mixings, Phys. Rev. Lett. 115 (2015) 181601 [arXiv:1503.01015] [INSPIRE].

[80] P. Adshead and M. Wyman, Gauge-flation trajectories in Chromo-Natural Inflation, Phys. Rev. D 86 (2012) 043530 [arXiv: 1203.2264] [INSPIRE].

[81] K. Choi, H. Kim and S. Yun, Natural inflation with multiple sub-Planckian axions, Phys. Rev. D 90 (2014) 023545 [arXiv: 1404.6209] [InSPIRE].

[82] R. Kappl, S. Krippendorf and H.P. Nilles, Aligned Natural Inflation: Monodromies of two Axions, Phys. Lett. B 737 (2014) 124 [arXiv:1404.7127] [InSPIRE].

[83] Y. Bai and B.A. Stefanek, Natural millicharged inflation, Phys. Rev. D 91 (2015) 096012 [arXiv: 1405.6720] [INSPIRE].

[84] A. de la Fuente, P. Saraswat and R. Sundrum, Natural Inflation and Quantum Gravity, Phys. Rev. Lett. 114 (2015) 151303 [arXiv:1412.3457] [InSPIRE].

[85] R. Coy, M. Frigerio and M. Ibe, Dynamical Clockwork Axions, JHEP 10 (2017) 002 [arXiv: 1706. 04529] [INSPIRE].

[86] K. Freese, J.A. Frieman and A.V. Olinto, Natural inflation with pseudo-Nambu-Goldstone bosons, Phys. Rev. Lett. 65 (1990) 3233 [INSPIRE].

[87] Planck collaboration, P.A.R. Ade et al., Planck 2015 results. XX. Constraints on inflation, Astron. Astrophys. 594 (2016) A20 [arXiv:1502.02114] [INSPIRE].

[88] N. Barnaby and M. Peloso, Large NonGaussianity in Axion Inflation, Phys. Rev. Lett. 106 (2011) 181301 [arXiv:1011.1500] [INSPIRE].

[89] N. Barnaby, R. Namba and M. Peloso, Phenomenology of a Pseudo-Scalar Inflaton: Naturally Large NonGaussianity, JCAP 04 (2011) 009 [arXiv:1102.4333] [INSPIRE].

[90] Planck collaboration, P.A.R. Ade et al., Planck 2015 results. XVII. Constraints on primordial non-Gaussianity, Astron. Astrophys. 594 (2016) A17 [arXiv:1502.01592] [INSPIRE].

[91] R.Z. Ferreira and A. Notari, Thermalized axion inflation: natural and monomial inflation with small $r$, Phys. Rev. D 97 (2018) 063528 [arXiv:1711.07483] [INSPIRE].

[92] P. Adshead, E. Martinec, E.I. Sfakianakis and M. Wyman, Higgsed Chromo-Natural Inflation, JHEP 12 (2016) 137 [arXiv: 1609.04025] [INSPIRE].

[93] T. Fujita, R. Namba and Y. Tada, Does the detection of primordial gravitational waves exclude low energy inflation?, Phys. Lett. B 778 (2018) 17 [arXiv:1705. 01533] [InSPIRE].

[94] E. McDonough and S. Alexander, Observable Chiral Gravitational Waves from Inflation in String Theory, arXiv:1806.05684 [INSPIRE].

[95] B. Heidenreich, M. Reece and T. Rudelius, The Weak Gravity Conjecture and Emergence from an Ultraviolet Cutoff, Eur. Phys. J. C 78 (2018) 337 [arXiv:1712.01868] [InSPIRE].

[96] A. Maleknejad and M.M. Sheikh-Jabbari, Gauge-flation: Inflation From Non-Abelian Gauge Fields, Phys. Lett. B 723 (2013) 224 [arXiv:1102.1513] [INSPIRE].

[97] M.M. Sheikh-Jabbari, Gauge-flation Vs Chromo-Natural Inflation, Phys. Lett. B 717 (2012) 6 [arXiv: 1203.2265] [INSPIRE].

[98] E. Dimastrogiovanni and M. Peloso, Stability analysis of chromo-natural inflation and possible evasion of Lyth's bound, Phys. Rev. D 87 (2013) 103501 [arXiv:1212.5184] [INSPIRE]. 
[99] A. Papageorgiou, M. Peloso and C. Unal, Nonlinear perturbations from the coupling of the inflaton to a non-Abelian gauge field, with a focus on Chromo-Natural Inflation, JCAP 09 (2018) 030 [arXiv: 1806.08313] [INSPIRE].

[100] B. Heidenreich, M. Reece and T. Rudelius, Sharpening the Weak Gravity Conjecture with Dimensional Reduction, JHEP 02 (2016) 140 [arXiv: 1509.06374] [INSPIRE].

[101] B. Heidenreich, M. Reece and T. Rudelius, Evidence for a sublattice weak gravity conjecture, JHEP 08 (2017) 025 [arXiv: 1606.08437] [INSPIRE].

[102] M. Montero, G. Shiu and P. Soler, The Weak Gravity Conjecture in three dimensions, JHEP 10 (2016) 159 [arXiv: 1606. 08438] [inSPIRE].

[103] S. Andriolo, D. Junghans, T. Noumi and G. Shiu, A Tower Weak Gravity Conjecture from Infrared Consistency, Fortsch. Phys. 66 (2018) 1800020 [arXiv: 1802.04287] [INSPIRE]. 